Freiburg-THEP 00/16

TTP 00-26

hep-ph/0011373

November 2000

\title{
Three-Loop Leading Top Mass Contributions to the $\rho$ Parameter
}

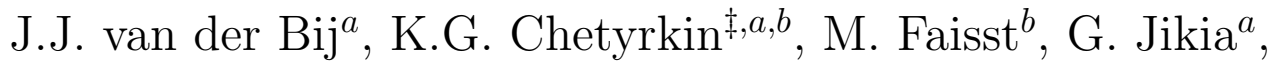 \\ and T. Seidensticker ${ }^{b}$
}

\author{
${ }^{a}$ Fakultät für Physik, \\ Albert-Ludwigs-Universität Freiburg, D-79104 Freiburg, Germany \\ ${ }^{b}$ Institut für Theoretische Teilchenphysik, \\ Universität Karlsruhe, D-76128 Karlsruhe, Germany
}

\begin{abstract}
We present analytical results for the leading contributions of the top quark to the electroweak $\rho$ parameter at order $G_{F}^{3} M_{t}^{6}$ and $G_{F}^{2} M_{t}^{4} \alpha_{s}$. The Higgs boson and the gauge bosons are taken to be massless in this limit. The correction of order $G_{F}^{3} M_{t}^{6}$ is found to be sizeable in comparison to the the leading two-loop $\mathcal{O}\left(G_{F}^{2} M_{t}^{4}\right)$ correction, however it is much smaller than the subleading $\mathcal{O}\left(G_{F}^{2} M_{t}^{2} M_{Z}^{2}\right)$ correction.
\end{abstract}

\section{Introduction}

The standard model of elementary particle physics has been in agreement with all experiments performed till date. The one ingredient that has not been found yet is the Higgs boson. Direct searches at LEP200 have been able to put a lower limit of $113 \mathrm{GeV}$ on its mass [1]. As the presence of the Higgs boson is necessary for the renormalizability of the theory, radiative processes calculated without taking into account the graphs containing virtual Higgs bosons would be infinite. Therefore radiative corrections should grow with the Higgs-boson mass. It is a well-known effect, sometimes called Veltman's theorem [2], that such corrections to low-energy parameters grow logarithmically with the Higgs mass. Therefore precision tests as performed at LEP can use the existing data to put bounds on the Higgs mass. An analysis of the data indicates that the Higgs boson should be light $(<170 \mathrm{GeV})$ [3] $]$. Therefore the Higgs boson should be found at the next generation of

\footnotetext{
${ }^{\ddagger}$ Permanent address: Institute for Nuclear Research, Russian Academy of Sciences, 60th October Anniversary Prospect 7a, Moscow 117312, Russia.
} 
colliders. However there is some lingering doubt as to the unavoidability of this conclusion. On the one hand there is a slight discrepancy between the leptonic and the hadronic data in the precision measurements. Taking the leptonic data only, the preferred value of the Higgs boson mass would be so light as to almost rule out the standard model already. On the other hand one should not change the model too much, as one can get rather large effects quickly, for instance a fourth generation appears to be definitely ruled out. The one option that is not quite under theoretical control is the case of strong interactions, which corresponds to a naively very heavy Higgs boson $\left(M_{H} \geq 1 \mathrm{TeV}\right)$. In this case higher order corrections could be large and cancel the leading order $\ln \left(M_{H}\right)$ effects, ultimately giving similar corrections as a light Higgs boson. Calculations at the two-loop order have been performed and actually give a negligeable effect [4]. The reason for this has been traced back to some apparently accidental cancellations between the coefficients in the Higgs mass dependent terms [5]. These cancellations appear to be typical for the low-energy vector boson-propagator and triple-vector boson interactions only [1]. Corrections to e.g. the fourvector boson interactions [6] or the Higgs-propagator [7] do not exhibit such cancellations. Therefore it may be possible that the full effect of the strong interactions shows up only at the three-loop level for the precision parameters at LEP. It is therefore imperative to push the calculations towards the three-loop order.

Of course, to determine the Higgs mass effects it is necessary to also have the effects of the other particles under very precise control, as these may be dominating. A particularly important parameter here is the so-called $\rho$ parameter which measures the relative strengths of the neutral and charged currents. At the tree-level this parameter is one, due to a residual $\mathrm{SU}(2)$, so-called custodial symmetry. This symmetry gets violated by the hyper-charge and by mass-splittings within doublets. Because the mass-splittings give contributions proportional to the square of the masses, the dominating effect is the contribution of the top quark $\left(\Delta \rho \approx G_{F} M_{t}^{2}\right)$ [8]. The Higgs mass dependence is only logarithmic and proportional to the hypercharge coupling $\left(\Delta \rho \approx g_{Y}^{2} \ln \left(M_{H} / M_{W}\right)\right)$ [9]. Since the Higgs mass dependent effect is sub-dominant, it could be masked by higher loop effects coming from the top quark. Therefore the higher loop top mass dependent corrections have to be calculated. As the top-Yukawa coupling is not very small, one should check whether perturbation theory in the Yukawa coupling is actually convergent. Known corrections at the two-loop level are the leading $\left(G_{F}^{2} M_{t}^{4}\right)$ and sub-leading $\left(g_{\text {weak }}^{2} G_{F} M_{t}^{2}\right)$ contributions. Numerically it was found that the subleading corrections are larger than the leading ones [10]. This naturally brings up the question whether the three-loop effect could also be large. There is actually an extra enhancement of the three-loop correction, because there is a term behaving like $n_{c}^{2}$ ( $n_{c}$ is the number of colors), whereas both one and two-loop corrections behave like $n_{c}$. If indeed the three-loop correction would be comparable to the two-loop one, one should consider whether there is a way to resum at least the leading order terms, maybe by a Borel summation. Such a resummation is not always possible, as one would need an alternating sign in the series. So independent of its direct phenomenological implications it is important to know the three-loop heavy-top correction to $\Delta \rho$, since it would give us a handle on the nature of perturbation theory in quantum field theory. Not very many quantities are known to such precision. 
As far as the calculation is concerned one must remark that a complete calculation at the three-loop level is impossible analytically. At best this could be done numerically. However the situation is somewhat simplified by taking the top mass much larger than $M_{W}$ or $M_{Z}$. In this case one can perform an expansion in the external momenta. The Feynman integrals that have to be calculated can then be reduced to vacuum graphs. These vacuum graphs are easier to calculate. At the two-loop level this program can be carried out till the end, as the vacuum-diagrams are known for arbitrary values of the masses. This way the leading and sub-leading corrections due to a heavy top quark have been calculated, including a full Higgs mass dependence [11, 12]. Numerically the Higgs mass dependence is quite sizeable. Unfortunately at the three-loop level vacuum diagrams are only known when there is one mass scale in the problem [13]. That is, every line in the graph is either massless or has a fixed mass. Mixed Higgs-top graphs can therefore only be calculated when the Higgs has the same mass as the top quark, or has mass zero. Also QCD corrections can be taken into account analytically. In this letter we consider the case that the top mass is much heavier than all other masses, effectively working in the $M_{H}=0$ limit. Within this limit we present the results of the calculation of the leading order $M_{t}^{6}$ and $\alpha_{s} M_{t}^{4}$ correction to $\Delta \rho$.

This letter is organized as follows: In the next Section we state our definition of the $\rho$ parameter and introduce our notations. Section 03 is devoted to a short discussion of the treatment of the diagrams and the renormalization. In Section 1 we present the results at order $G_{F}^{3} M_{t}^{6}$ and $G_{F}^{2} M_{t}^{4} \alpha_{s}$.

\section{Definition}

The $\rho$ parameter is usually defined by the ratio of the neutral and charged current coupling constants at zero momentum transfer:

$$
\rho=\frac{J_{N C}(0)}{J_{C C}(0)}=\frac{1}{1-\Delta \rho} .
$$

$J_{C C}(0)$ is given by the Fermi coupling constant $G_{F}$ determined from the $\mu$ decay rate whereas $J_{N C}(0)$ is measured by neutrino scattering on electrons or hadrons. This definition of the $\rho$ parameter is principally speaking not process independent, since the radiative corrections depend on the value of the hypercharge of the particles in the process. However the leading terms in the top mass are process independent.

The origin of the process dependence lies in the different hypercharge assignments of the particles that take part in the scattering cross-sections. As already mentioned in the introduction, the origin of a non-zero value of $\Delta \rho$ is due to the breaking of the custodial $\mathrm{SU}(2)$ symmetry. This breaking originates from either the hypercharge coupling or from the doublet mass-splittings. In leading order these effects are separate, but in higher order the effects of the Yukawa and the gauge couplings become intricately intertwined. At the twoloop level there is a leading $\mathcal{O}\left(M_{t}^{4}\right)$ correction, that is independent of the gauge-couplings completely. Even in the absence of the hypercharge field there is a subleading $\mathcal{O}\left(M_{t}^{2}\right)$ 
contribution. Finally the exact sub-leading term becomes a function of the hypercharge coupling, giving rise to a more complicated formula as a function of $\sin ^{2}\left(\theta_{W}\right)$ [10]. As the whole correction is also strongly dependent on the Higgs mass one should be careful in drawing conclusions from the large size of the subleading term compared to the leading term. This effect may be specific to the particular observable being calculated. In particular there appears to be an accidental cancellation between different coefficients in the leading term $\mathcal{O}\left(G_{F}^{2} M_{t}^{4}\right)$ to $\Delta \rho$ for $M_{H}=0$, which disappears for a larger Higgs mass and which is absent in the correction to $Z \rightarrow b \bar{b}$.

In leading order in the top mass contributions to $\rho$ stem from the transversal parts of the (unrenormalized) self energies of the exchanged vector bosons $W$ and $Z$ :

$$
\rho=\frac{1-\Pi_{T}^{W W}(0) / M_{W_{0}}^{2}}{1-\Pi_{T}^{Z Z}(0) / M_{Z_{0}}^{2}},
$$

where $M_{W_{0}} M_{Z_{0}}$ are the bare masses of the $W$ and $Z$ bosons respectively. In this form the results would appear infinite, but these infinities are canceled by the Higgs mass, top mass and $W$ boson mass renormalizations. Corrections from vertex and box diagrams always involve extra powers of the weak coupling constant $g_{\text {weak }}$ and are therefore suppressed by powers of $g_{\text {weak }} / g_{Y \text { Yuawa }}^{\text {top }}$, i.e. $M_{W} / M_{t}$.

A remark is here in order. Up to the two-loop level for the leading top mass effects the correction to $\rho$ can be expressed in a number of equivalent ways, i.e. in terms of $G_{F}^{2} M_{t}^{4}$ or $M_{t}^{4} / M_{W}^{4}$. At the three-loop level one must be more precise. At this level the difference between low-energy parameters and on-shell vector boson masses plays a role even in the leading $M_{t}$ effects. In the following we will express the final answer in terms of the on-shell top mass and the low-energy Fermi constant $G_{F}$ from $\mu$-decay.

\section{Treatment of diagrams and renormalization}

There are four major contributions to the three-loop $\rho$ parameter which we will discuss below. First, the three-loop self energy diagrams of the $W$ and $Z$ boson, of course. Second, the top quark mass renormalization at order $G_{F}^{2} M_{t}^{4}$ and $G_{F} M_{t}^{2} \alpha_{s}$. Third, the renormalization of the vacuum expectation value of the Higgs field and fourth, the Higgs mass renormalization. For all contributions we have limited ourselves to the leading top quark mass effects which simplifies the computation drastically.

As far as the self energy diagrams of the $W$ and $Z$ bosons are concerned we have chosen to compute an asymptotic expansion w.r.t. the external momentum since we are interested in the zero momentum transfer limit. The treatment of the Higgs mass has been done using two different approaches: First, an asymptotic expansion [14 w.r.t. the Higgs mass and second, setting the Higgs mass equal to zero right from the start. Both strategies lead to the same result as no Higgs mass dependence is present in the final results.

Throughout this computation we have used several computer programs and packages. The diagrams were generated using QGRAF [15] and the asymptotic expansion in limit of the small Higgs mass was performed by EXP [16]. The resulting three-loop integrals were 


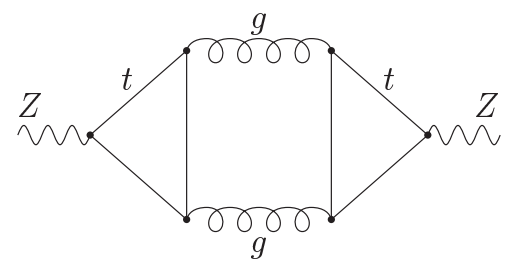

(a)

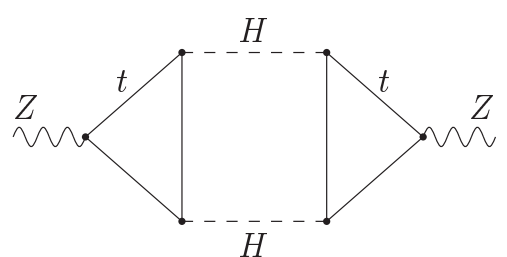

(b)

Figure 1: (a) A diagram for which the traces with an odd number of $\gamma_{5}$ matrixes do contribute; (b) a diagram which can be safely treated with the completely anticommutating $\gamma_{5}$.

evaluated with the help of the program packages MINCER 177 and MATAD [18 written in FORM [19]. The two-loop top quark mass renormalization constant requires the calculation of two-loop on-shell diagrams. We have used the packages TARCER [20] and ONSHELL2 [21] for the computation of these types of diagrams.

The renormalization of the vacuum expectation value of the Higgs field is fixed by the requirement of vanishing Higgs tadpoles. It also influences the Higgs mass renormalization and the mass renormalization of the Goldstone bosons. We need this renormalization constant up to two-loops and the computation of the tadpoles is straightforward. The mass renormalization of the Higgs field is required only up to one-loop order.

We have used the prescription of a completely anticommutating $\gamma_{5}$ in the evaluation of all traces. In particular, all traces with an odd number of $\gamma_{5}$ 's were set to zero. This treatment is completely justified if and only if every such diagram vanishes on kinematical grounds. For instance, obviously no problem could result from 2-point amplitudes with only one fermion loop: the number of available independent external momenta (one) and Lorentz indices (two if both legs are vector bosons) is not enough to form a completely ntisymmetric tensor $\epsilon_{\mu \nu \lambda \rho}$. The situation can be different only for singlet type 3-loop diagrams comprising two fermion traces (see Fig. 1).

Indeed, the trace occurring in one-loop vertex diagram with three vector external legs (Fig. 1(a)) can only be computed by using the 't Hooft-Veltman prescription for $\gamma_{5}$. Fortunately, in our case the only dangerous diagrams are those of Fig. 1(b). They contain at worst triangle subdiagrams comprising a trace of an odd number of $\gamma_{5}$ 's and depending on only three (not four!) independent external Lorentz vectors and indices (two independent external momenta and at maximum one index from a vector boson leg).

We have also successfully checked the Ward-Takahashi identities which relate the selfenergies of the gauge bosons to the non-diagonal self-energies of the gauge-to-Goldstone and the Goldstone boson self-energies. 


\section{Results}

We have reproduced the one- and two-loop results for the $\rho$ parameter at orders $G_{F} M_{t}^{2}$, $G_{F}^{2} M_{t}^{4}$ and $G_{F} M_{t}^{2} \alpha_{s}$ that can be found in [8, 22, 23]:

$$
\begin{aligned}
\Delta \rho^{\left(1, M_{t}^{2}\right)} & =n_{c} X_{t}, \\
\Delta \rho^{\left(2, M_{t}^{4}\right)} & =n_{c} X_{t}^{2}\left(19-12 \zeta_{2}\right), \\
\Delta \rho^{\left(2, \alpha_{s} M_{t}^{2}\right)} & =n_{c} X_{t} \frac{\alpha_{s}}{\pi} C_{F}\left(-\frac{1}{2}-\zeta_{2}\right) .
\end{aligned}
$$

$X_{t}$ is defined by $\left(M_{t}\right.$ is the top quark pole mass)

$$
X_{t}=\frac{G_{F} M_{t}^{2}}{8 \sqrt{2} \pi^{2}}
$$

and $n_{c}$ represents the number of colours. $\zeta_{n}$ is used for the values of the Riemann $\zeta$-function at $n, \zeta(n)$, giving e.g. $\zeta_{2}=\pi^{2} / 6 . C_{F}$ is equal to $4 / 3$ for the $\mathrm{SU}(3)$ colour group.

For the light Higgs boson the subleading two-loop $\mathcal{O}\left(G_{F}^{2} M_{t}^{2} M_{Z}^{2}\right)$ corrections are much larger than the leading two-loop $\mathcal{O}\left(G_{F}^{2} M_{t}^{4}\right)$ term, which is actually suppressed by accidental cancellations [10]. So they should necessarily be taken into account. In contrast to the leading corrections the subleading $M_{t}^{2}$ two-loop corrections depend on the processes used to define the neutral and charged currents in Eq. (回). If the charged current coupling constant is defined through the $\mu$-decay and the neutral current coupling constant is defined through the $\nu_{\mu} e$ scattering, then the subleading two-loop corrections in the massless Higgs limit are given by

$$
\begin{aligned}
\Delta \rho^{\left(2, M_{t}^{2}\right)}= & n_{c} X_{t}^{2} \frac{M_{Z}^{2}}{M_{t}^{2}}\left[-\frac{11}{2}+\frac{3}{\hat{s}^{2}}+\frac{319 \hat{s}^{2}}{9}+6 \hat{c}^{2} I_{3}+\pi^{2}\left(-\frac{7}{3}-\frac{56 \hat{s}^{2}}{27}\right)\right. \\
& \left.+\left(7+\frac{3}{\hat{s}^{4}}-\frac{6}{\hat{s}^{2}}-4 \hat{s}^{2}\right) \ln \left(\hat{c}^{2}\right)+\left(21-16 \hat{s}^{2}\right) \ln \left(\frac{M_{Z}^{2}}{M_{t}^{2}}\right)\right],
\end{aligned}
$$

with $I_{3}$ representing the isospin of the electron target $\left(I_{3}=-1\right)$ [10]. $\hat{s}$ and $\hat{c}$ are abbreviations for the $\overline{\mathrm{MS}}$ quantities $\sin \hat{\theta}_{W}$ and $\cos \hat{\theta}_{W}$, respectively, given by $\hat{c}^{2} \approx c^{2}\left(1-n_{c} X_{t}\right)$ with the on-shell parameter $c^{2}=M_{W}^{2} / M_{Z}^{2}$. In the limit of vanishing hypercharge $(\hat{s}=0)$ the corresponding correction looks like

$$
\left.\Delta \rho^{\left(2, M_{t}^{2}\right)}\right|_{\hat{s}=0}=n_{c} X_{t}^{2} \frac{M_{Z}^{2}}{M_{t}^{2}}\left[-1-\frac{7 \pi^{2}}{3}+6 I_{3}+21 \ln \left(\frac{M_{Z}^{2}}{M_{t}^{2}}\right)\right] .
$$

Numerically the subleading corrections (7-8) are about 20 times larger than the leading two-loop correction (4) and are about $4 \%$ of the one-loop correction (3).

The three-loop results read

$$
\begin{aligned}
& \Delta \rho^{\left(3, M_{t}^{6}\right)}= \\
& \quad X_{t}^{3} n_{c}\left(68+729 S_{2}+36 D_{3}+96 \zeta_{2} \ln 2+6 \zeta_{2}-612 \zeta_{3}+324 \zeta_{4}-72 B_{4}\right) \\
& \quad+X_{t}^{3} n_{c}^{2}\left(-\frac{6572}{15}-\frac{4374}{5} S_{2}+\frac{1472}{15} \zeta_{2}+440 \zeta_{3}\right)
\end{aligned}
$$




\begin{tabular}{|c|c|c|c|c|c|c|}
\hline$X_{t}$ & $X_{t}^{2}$ & $X_{t}^{2} \frac{M_{Z}^{2}}{M_{t}^{2}}$ & $X_{t} \alpha_{s}$ & $X_{t}^{3}$ & $X_{t}^{2} \alpha_{s}$ & $X_{t} \alpha_{s}^{2}$ \\
\hline $9.6 \cdot 10^{-3}$ & $-2.3 \cdot 10^{-5}$ & $-4.1 \cdot 10^{-4}$ & $-9.5 \cdot 10^{-4}$ & $8.2 \cdot 10^{-6}$ & $1.0 \cdot 10^{-6}$ & $-1.7 \cdot 10^{-4}$ \\
\hline
\end{tabular}

Table 1: Size of different contributions to the $\rho$ parameter according to Eq. (13). The numbers are obtained with $\alpha_{s}\left(M_{t}\right)=0.109$.

and

$$
\begin{aligned}
& \Delta \rho^{\left(3, M_{t}^{4} \alpha_{s}\right)}= \\
& \quad X_{t}^{2} n_{c} \frac{\alpha_{s}}{\pi} C_{F}\left(\frac{185}{3}+\frac{729}{4} S_{2}-48 \zeta_{2} \ln 2-\frac{151}{6} \zeta_{2}+29 \zeta_{3}-24 \zeta_{4}+12 B_{4}\right) .
\end{aligned}
$$

The constants $S_{2}, D_{3}$, and $B_{4}$ are given by (see, e.g. [18])

$$
\begin{aligned}
S_{2} & =\frac{4}{9 \sqrt{3}} \mathrm{Cl}_{2}\left(\frac{\pi}{3}\right)=0.260434 \ldots \\
D_{3} & =6 \zeta_{3}-\frac{15}{4} \zeta_{4}-6\left[\mathrm{Cl}_{2}\left(\frac{\pi}{3}\right)\right]^{2}=-3.027009 \ldots \\
B_{4} & =16 \operatorname{Li}_{4}\left(\frac{1}{2}\right)-4 \zeta_{2} \ln ^{2} 2+\frac{2}{3} \ln ^{4} 2-\frac{13}{2} \zeta_{4}=-1.762800 \ldots
\end{aligned}
$$

with $\mathrm{Cl}_{2}(\pi / 3)=\operatorname{Im}\left[\mathrm{Li}_{2}\left(e^{i \pi / 3}\right)\right] . \mathrm{Li}_{2}$ and $\mathrm{Li}_{4}$ represent the Di- and Quadrilogarithm, respectively.

Numerically, we find

$$
\begin{aligned}
\Delta \rho= & n_{c} X_{t}-0.7392 n_{c} X_{t}^{2}-X_{t}^{2} \frac{M_{Z}^{2}}{M_{t}^{2}} n_{c}\left(30.029-21 \ln \left(\frac{M_{Z}^{2}}{M_{t}^{2}}\right)\right) \\
& +n_{c}\left(10.1466+24.3669 n_{c}\right) X_{t}^{3} \\
& +\frac{\alpha_{s}}{\pi} n_{c}\left(-2.8599 X_{t}+0.9798 X_{t}^{2}\right)+\cdots
\end{aligned}
$$

and, for $n_{c}=3$,

$$
\begin{aligned}
\Delta \rho= & 3 X_{t}-2.2176 X_{t}^{2}-X_{t}^{2} \frac{M_{Z}^{2}}{M_{t}^{2}}\left(90.1-63 \ln \left(\frac{M_{Z}^{2}}{M_{t}^{2}}\right)\right)+249.74 X_{t}^{3} \\
& +\frac{\alpha_{s}}{\pi}\left(-8.5797 X_{t}+2.9394 X_{t}^{2}\right) \\
& -43.782\left(\frac{\alpha_{s}}{\pi}\right)^{2} X_{t}+\cdots
\end{aligned}
$$

Here the subleading two-loop correction is given again for the process of $\nu_{\mu} e$ scattering and in order to see the pure effect of the top quark mass on $\Delta \rho$ the value $\hat{s}=0$ was taken. For the realistic value of $\hat{s}^{2}=0.2311$ the subleading two-loop corrections are 
$-X_{t}^{2} M_{Z}^{2} / M_{t}^{2}\left(78.7-51.9 \ln \left(M_{Z}^{2} / M_{t}^{2}\right)\right)$. For completeness we have also added to the r.h.s. of (13) the well known term of order $G_{F} M_{t}^{2} \alpha_{s}^{2}$ [24].

The size of the various terms in Eq. (13) is shown in Table 1. A few remarks are in order. First, the coefficients of the perturbative series are alternating for both pure electroweak and mixed electroweak/strong corrections. Second, the size of the three-loop electroweak coefficient is quite large and results in a correction to the leading two-loop contribution of approximately $36 \%$ (for $X_{t} \approx 3.2 \cdot 10^{-3}$ ). The size of the three-loop correction relative to the much larger subleading two-loop $M_{t}^{2}$ corrections (7/8) is however about $2 \%$. The mixed $\mathcal{O}\left(G_{F}^{2} M_{t}^{4} \alpha_{s}\right)$ term is numerically less than the purely electroweak $\mathcal{O}\left(G_{F}^{3} M_{t}^{6}\right)$ one by almost an order of magnitude. Finally, the major part of the $\mathcal{O}\left(X_{t}^{3}\right)$ contribution to the $\rho$ parameter is given by the leading term in the large $n_{c}$ approximation.

With respect to the question of the convergence of perturbation theory in the Yukawacoupling $X_{t}$ the results give only limited information. Because of the dependence on $n_{c}$ one can actually study two different limits, selecting different subsets of graphs. The first possibility is to take the graphs with the largest number of fermion-loops into account, which is one less than the number of loops, starting at the two-loop level. Formally one can project out these graphs by taking $X_{t} \approx 1 / n_{c}$ and take the limit $n_{c} \rightarrow \infty$. With the exception of one-loop all other loops are of the same order. One can thus only meaningfully compare the two-loop with the three-loop level. These become equal for $n_{c} X_{t} \approx 0.03$, corresponding to $M_{t} \approx 300 \mathrm{GeV}$. This would naively imply an early breakdown of perturbation theory. Such a conclusion however would be premature, as it is known that the two-loop $\rho$ parameter has a large cancellation between the different terms, that have not been observed for other quantities.

The other limit is taking into account only graphs with one fermion-loop, which one can describe formally by taking the limit $n_{c} \rightarrow 0$. Here one has three terms in the perturbation expansion available and can therefore form the [1,1]-Padé approximant. This gives

$$
\Delta \rho=n_{c} X_{t} \frac{1+12.987 X_{t}}{1+13.7265 X_{t}} .
$$

For a large top mass $\left(X_{t} \rightarrow \infty\right)$ this would imply a saturation of the radiative correction

$$
\Delta \rho=0.946 \Delta \rho_{1-\text { loop }}
$$

However also this early saturation could be an artefact, coming from the anomalously small two-loop leading term. All in all one gains little insight here without having a way to sum high-loop graphs. Also a comparison with other quantities would be necessary as well as a study of the Higgs mass dependence.

\section{Conclusion}

We have analytically computed the leading contributions of the top quark to the electroweak $\rho$ parameter at order $G_{F}^{3} M_{t}^{6}$ and $G_{F}^{2} M_{t}^{2} \alpha_{s}$. The correction of order $G_{F}^{3} M_{t}^{6}$ is 
sizeable in comparison to the leading two-loop correction. As we have mentioned in the Introduction the three-loop leading correction in principle could have happened to be of the same order of magnitude as the subleading $G_{F}^{2} M_{t}^{2} M_{Z}^{2}$ two-loop correction. We have found, however, that in fact it is still much smaller than the subleading two-loop one. Thereby we have significantly reduced the theoretical uncertainty of the results of electroweak higher order calculations coming from the enhanced three-loop large top quark mass contributions.

\section{Acknowledgements}

This work was supported by the Graduiertenkolleg "Elementarteilchenphysik an Beschleunigern" and the DFG-Forschergruppe "Quantenfeldtheorie, Computeralgebra und MonteCarlo-Simulation" ( contract FOR 264/2-1). It was also supported by the European Union under contract HPRN-CT-2000-00149.

The authors would like to thank J.H. Kühn for useful discussions and advice. We are grateful to M.Yu. Kalmykov for providing us with an updated version of the package ONSHELL2.

\section{References}

[1] P. Igo-Kemenes, ICHEP2000, Osaka 2000.

[2] M.J.G. Veltman, Act. Phys. Pol. B 8 (1977) 475.

[3] A. Gurtu, ICHEP2000, Osaka 2000.

[4] J.J. van der Bij and M.J.G. Veltman, Nucl. Phys. B 231 (1984) 205; J.J. van der Bij, Nucl. Phys. B 248 (1984) 141, Nucl. Phys. B 255 (1985) 648.

[5] R. Akhoury, J.J. van der Bij, and H. Wang, preprint hep-ph/0010187.

[6] V. Borodulin and G. Jikia, Nucl. Phys. B 520 (1998) 31;

G. Jikia, Act. Phys. Pol. B 29 (1998) 2719.

[7] A. Ghinculov and J.J. van der Bij, Nucl. Phys. B 436 (1995) 30.

[8] M.J.G. Veltman, Nucl. Phys. B 123 (1977) 89.

[9] A.C. Longhitano, Phys. Rev. D 22 (1980) 1166.

[10] G. Degrassi, S. Fanchiotti, F. Feruglio, P. Gambino, and A. Vicini, Phys. Lett. B 350 (1995) 75;

G. Degrassi, P. Gambino, and A. Vicini, Phys. Lett. B 383 (1996) 219. 
[11] R. Barbieri, M. Beccaria, P. Giafaloni, G. Curci and A. Viceré, Phys. Lett. B 288 (1992) 95, E: Phys. Lett. B 312 (1993) 511; Nucl. Phys. B 409 (1993) 105.

[12] J. Fleischer, F. Jegerlehner and O. V. Tarasov, Phys. Rev. D 51 (1995) 3820.

[13] D.J. Broadhurst, Eur. Phys. J. C8, 1999, 311.

[14] see e.g.,V.A. Smirnov, Mod. Phys. Lett. A 10 (1995) 1485, and references therein.

[15] P. Nogueira, J. Comp. Phys. 105 (1993) 279.

[16] T. Seidensticker, Diploma thesis (University of Karlsruhe, 1998), unpublished.

[17] S.A. Larin, F.V. Tkachov, and J.A.M. Vermaseren, preprint NIKHEF-H/91-18 (1991).

[18] M. Steinhauser, preprint hep-ph/0009029; see also: PhD thesis (University of Karlsruhe), Shaker Verlag, Aachen, 1996.

[19] J.A.M. Vermaseren, Symbolic Manipulation with FORM, CAN (1991).

[20] R. Mertig and R. Scharf, Comp. Phys. Commun. 111 (1998) 265.

[21] J. Fleischer and M.Yu. Kalmykov, Comp. Phys. Commun. 128 (2000) 531.

[22] A. Djouadi and C. Verzegnassi, Phys. Lett. B 195 (1990) 265;

A. Djouadi, Nuovo Cim. 100A (1988) 357.

[23] J.J. van der Bij and F. Hoogeveen, Nucl. Phys. B 283 (1987) 477.

[24] L. Avdeev, J. Fleischer, S.M. Mikhailov, and O. Tarasov, Phys. Lett. B 336 (1994) 560; E: Phys. Lett. B 349 (1995) 597;

K.G. Chetyrkin, J.H. Kühn, and M. Steinhauser, Phys. Lett. B 351 (1995) 331. 